\title{
Strong-Coupling Constant with Flavor Thresholds at Five Loops in the $\overline{\mathrm{MS}}$ Scheme
}

\author{
B.A. Kniehl, A.V. Kotikov* A.I. Onishchenko ${ }^{\dagger}$ and O.L. Veretint \\ II. Institut für Theoretische Physik, \\ Universität Hamburg, 22761 Hamburg, Germany
}

(Dated: June 5, 2006)

\begin{abstract}
We present in analytic form the matching conditions for the strong-coupling constant $\alpha_{s}^{\left(n_{f}\right)}(\mu)$ at the flavor thresholds to four loops in the modified minimal-subtraction scheme. Taking into account the present knowledge on the coefficient $\beta_{4}$ of the Callan-Symanzik beta function of quantum chromo-dynamics, we thus derive a five-loop formula for $\alpha_{s}^{\left(n_{f}\right)}(\mu)$ together with appropriate relationships between the asymptotic scale parameters $\Lambda^{\left(n_{f}\right)}$ for different numbers of flavors $n_{f}$.

PACS numbers: 11.10.Hi, 11.15.Me, 12.38.-t, 12.38.Bx
\end{abstract}

\footnotetext{
*Also at Bogolyubov Laboratory for Theoretical Physics, JINR, 141980 Dubna (Moscow region), Russia. ${ }^{\dagger}$ Also at Theoretical Physics Department, Petersburg Nuclear Physics Institute, Orlova Roscha, 188300 Gatchina, Russia.

${ }^{\ddagger}$ Also at Petrozavodsk State University, 185910 Petrozavodsk, Karelia, Russia.
} 
The strong-coupling constant $\alpha_{s}^{\left(n_{f}\right)}(\mu)=g_{s}^{2} /(4 \pi)$, where $g_{s}$ is the gauge coupling of quantum chromo-dynamics (QCD), is a fundamental parameter of the standard model of elementary particle physics; its value $\alpha_{s}^{(5)}\left(M_{Z}\right)$ is listed among the constants of nature in the Review of Particle Physics [1]. Here, $\mu$ is the renormalization scale, and $n_{f}$ is the number of active quark flavors $q$, with mass $m_{q} \ll \mu$. The $\mu$ dependence of $\alpha_{s}^{\left(n_{f}\right)}(\mu)$ is controlled by the Callan-Symanzik beta function of QCD,

$$
\begin{aligned}
\mu^{2} \frac{d}{d \mu^{2}} \frac{\alpha_{s}^{\left(n_{f}\right)}(\mu)}{\pi} & =\beta^{\left(n_{f}\right)}\left(\frac{\alpha_{s}^{\left(n_{f}\right)}(\mu)}{\pi}\right) \\
& =-\sum_{N=0}^{\infty} \beta_{N}^{\left(n_{f}\right)}\left(\frac{\alpha_{s}^{\left(n_{f}\right)}(\mu)}{\pi}\right)^{N+2} .
\end{aligned}
$$

The calculation of the one-loop coefficient $\beta_{0}^{\left(n_{f}\right)}$ about 33 years ago [2] has led to the discovery of asymptotic freedom and to the establishment of QCD as the theory of strong interactions, an achievement that was awarded by the 2004 Nobel Prize in Physics. In the class of schemes where the beta function is mass independent, which includes the minimal-subtraction (MS) schemes of dimensional regularization [3], $\beta_{0}^{\left(n_{f}\right)}$ and $\beta_{1}^{\left(n_{f}\right)}$ [4] are universal. The results for $\beta_{2}^{\left(n_{f}\right)}[\underline{5}]$ and $\beta_{3}^{\left(n_{f}\right)}$ [6] are available in the modified MS $(\overline{\mathrm{MS}})$ scheme [7]. As for $\beta_{4}^{\left(n_{f}\right)}$, the term proportional to $n_{f}^{4}$,

$$
\beta_{4}^{\left(n_{f}\right)}=\left[\frac{1205}{2985984}-\frac{19}{10368} \zeta(3)\right] n_{f}^{4}+O\left(n_{f}^{3}\right)
$$

where $\zeta$ is Riemann's zeta function, was found in the large- $n_{f}$ expansion [8], while the residual terms, of $O\left(n_{f}^{3}\right)$ and below, are presently unknown. However, the latter were estimated by an educated guess, through weighted asymptotic Padé approximant predictions (WAPAP's), which are improved by including asymptotic corrections with respect to the usual Padé approximants and performing a weighted average over negative values of $n_{f}[9]$. In the case of $\beta_{3}^{\left(n_{f}\right)}$, leaving aside the quartic Casimir terms, which appear there for the first time, the WAPAP's approximate the exact coefficients of $n_{f}^{n}$ with $n=0,1,2$ amazingly well, at the one-percent level. One may thus expect that the WAPAP's for $\beta_{4}^{\left(n_{f}\right)}$ work similarly well, except for the quartic Casimir terms, which cannot be predicted quite as reliably. For the reader's convenience, $\beta_{N}^{\left(n_{f}\right)}(N=0, \ldots, 4)$ are listed for the $n_{f}$ values of practical interest in Table 【.

In MS-like renormalization schemes, the Appelquist-Carazzone decoupling theorem 10] does not in general apply to quantities that do not represent physical observables, such as 
TABLE I: $\overline{\mathrm{MS}}$ values of $\beta_{N}^{\left(n_{f}\right)}$ for variable $n_{f} \cdot \beta_{4}^{\left(n_{f}\right)}$ is estimated by WAPAP's with quartic Casimir terms omitted [9].

\begin{tabular}{|c|c|c|c|c|c|}
\hline$n_{f}$ & $\beta_{0}^{\left(n_{f}\right)}$ & $\beta_{1}^{\left(n_{f}\right)}$ & $\beta_{2}^{\left(n_{f}\right)}$ & $\beta_{3}^{\left(n_{f}\right)}$ & $\beta_{4}^{\left(n_{f}\right)}$ \\
\hline 3 & $\frac{9}{4}$ & 4 & $\frac{3863}{384}$ & $\frac{445}{32} \zeta(3)+\frac{140599}{4608}$ & 162 \\
\hline 4 & $\frac{25}{12}$ & $\frac{77}{24}$ & $\frac{21943}{3456}$ & $\frac{78535}{5184} \zeta(3)+\frac{4918247}{373248}$ & 119 \\
\hline 5 & $\frac{23}{12}$ & $\frac{29}{12}$ & $\frac{9769}{3456}$ & $\frac{11027}{648} \zeta(3)-\frac{598391}{373248}$ & 107 \\
\hline 6 & $\frac{7}{4}$ & $\frac{13}{8}$ & $-\frac{65}{128}$ & $\frac{11237}{576} \zeta(3)-\frac{63559}{4608}$ & 124 \\
\hline
\end{tabular}

beta functions or coupling constants, i.e., quarks with mass $m_{q} \gg \mu$ do not automatically decouple. The standard procedure to circumvent this problem is to render decoupling explicit by using the language of effective field theory. As an idealized situation, consider QCD with $n_{l}=n_{f}-1$ massless quark flavors and one heavy flavor $h$, with mass $m_{h} \gg \mu$. Then, one constructs an effective $n_{l}$-flavor theory by requiring consistency with the full $n_{f}$-flavor theory at the heavy-quark threshold $\bar{\mu}=\mathcal{O}\left(m_{h}\right)$. This leads to a nontrivial matching condition between the couplings of the two theories. Although, $\alpha_{s}^{\left(n_{l}\right)}\left(m_{h}\right)=\alpha_{s}^{\left(n_{f}\right)}\left(m_{h}\right)$ at leading and next-to-leading orders, this relationship does not generally hold at higher orders in the $\overline{\mathrm{MS}}$ scheme, i.e., $\alpha_{s}^{\left(n_{f}\right)}(\mu)$ starts to exhibit finite discontinuities at the flavor thresholds. If the $\mu$ evolution of $\alpha_{s}^{\left(n_{f}\right)}(\mu)$ is to be performed at $N+1$ loops, i.e., with the highest coefficient in Eq. (II) being $\beta_{N}^{\left(n_{f}\right)}$, then consistency requires that the matching conditions be implemented in terms of $N$-loop formulae. Then, the residual $\mu$ dependence of physical observables will be of order $N+2$. The QCD matching conditions at the flavor thresholds to two [1]] and three [12] loops are known in analytical form; they are routinely used in the literature and even copied to the Review of Particle Physics [1]. Recently, the four-loop result was found, in semi-analytical form [13]. In fact, the most intricate four-loop tadpole master integrals involving one non-vanishing mass among the basic set that enters any such calculation could so far only be computed numerically, with limited precision [13, 14, 15]. It is the purpose of this Letter, to overcome this bottle-neck by presenting the four-loop matching condition for $\alpha_{s}^{\left(n_{f}\right)}(\mu)$ entirely in terms of elementary transcendental numbers. This requires the analytic evaluation of the massive four-loop tadpole diagram that is called $X_{0}$ or $T_{91}$ in the recent literature [13]. Together with the results of Ref. [16], we thus enhance the knowledge of the 
basic set of massive four-loop tadpole master integrals in analytic form.

Prior to explaining the core of this analysis and presenting our analytic result for the four-loop matching condition for $\alpha_{s}^{\left(n_{f}\right)}(\mu)$, we derive the five-loop formula for this coupling for fixed value of $n_{f}$. In order to simplify the notation, we introduce the couplant $a^{\left(n_{f}\right)}(\mu)=$ $\alpha_{s}^{\left(n_{f}\right)}(\mu) / \pi$ and omit the labels $\mu$ and $n_{f}$ wherever confusion is impossible. Integrating Eq. (11) leads to

$$
\begin{aligned}
\ln \frac{\mu^{2}}{\Lambda^{2}} & =\int \frac{d a}{\beta(a)}=\frac{1}{\beta_{0}}\left[\frac{1}{a}+b_{1} \ln a+a\left(-b_{1}^{2}+b_{2}\right)\right. \\
+ & a^{2}\left(\frac{b_{1}^{3}}{2}-b_{1} b_{2}+\frac{b_{3}}{2}\right)+a^{3}\left(-\frac{b_{1}^{4}}{3}+b_{1}^{2} b_{2}-\frac{b_{2}^{2}}{3}\right. \\
& \left.\left.-\frac{2}{3} b_{1} b_{3}+\frac{b_{4}}{3}\right)+O\left(a^{4}\right)\right]+C,
\end{aligned}
$$

where $b_{N}=\beta_{N} / \beta_{0}(N=1, \ldots, 4), \Lambda$ is the so-called asymptotic scale parameter, and $C$ is an arbitrary constant. The second equality in Eq. (3) is obtained by expanding the integrand. The conventional $\overline{\mathrm{MS}}$ definition of $\Lambda$, which we adopt, corresponds to choosing $C=\left(b_{1} / \beta_{0}\right) \ln \beta_{0}$ [7, 17]. Iteratively solving Eq. (3) yields, with $L=\ln \left(\mu^{2} / \Lambda^{2}\right)$,

$$
\begin{aligned}
a= & \frac{1}{\beta_{0} L}-\frac{b_{1} \ln L}{\left(\beta_{0} L\right)^{2}}+\frac{1}{\left(\beta_{0} L\right)^{3}}\left[b_{1}^{2}\left(\ln ^{2} L-\ln L-1\right)+b_{2}\right] \\
& +\frac{1}{\left(\beta_{0} L\right)^{4}}\left[b_{1}^{3}\left(-\ln ^{3} L+\frac{5}{2} \ln ^{2} L+2 \ln L-\frac{1}{2}\right)\right. \\
& \left.-3 b_{1} b_{2} \ln L+\frac{b_{3}}{2}\right]+\frac{1}{\left(\beta_{0} L\right)^{5}}\left[b _ { 1 } ^ { 4 } \left(\ln ^{4} L-\frac{13}{3} \ln ^{3} L\right.\right. \\
& \left.-\frac{3}{2} \ln ^{2} L+4 \ln L+\frac{7}{6}\right)+3 b_{1}^{2} b_{2}\left(2 \ln ^{2} L-\ln L-1\right) \\
& \left.-b_{1} b_{3}\left(2 \ln L+\frac{1}{6}\right)+\frac{5}{3} b_{2}^{2}+\frac{b_{4}}{3}\right]+O\left(\frac{1}{L^{6}}\right) .
\end{aligned}
$$

The particular choice of $C$ [7, 17] in Eq. (3) is predicated on the grounds that it suppresses the appearance of a term proportional to (const./ $\left.L^{2}\right)$ in Eq. (4).

We now turn to the analytic evaluation of the four-loop matching condition for $\alpha_{s}^{\left(n_{f}\right)}(\mu)$ at the flavor thresholds. The underlying formalism was comprehensively explained in Refs. 11, 12], and most of the technical issues related to its application at four loops were already discussed in Ref. [13]. For lack of space, we thus concentrate here on the missing link of this analysis beyond the scope of Ref. 13], namely the analytic evaluation of the massive four-loop tadpole diagram $X_{0}$, which is depicted in Fig. 1(a). This task may be simplified by noticing that $X_{0}$ does not represent a master integral, but may be reduced to simpler 


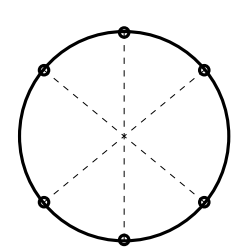

(a)

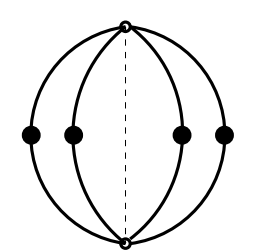

(b)

FIG. 1: Four-loop tadpole diagrams (a) $X_{0}$ and (b) $J_{0}$. Dashed and solid lines represent massless and massive propagators; a dot on a line duplicates that propagator.

integrals with less lines, all of which are analytically known [14], some for a short time only [16], except for the one $\left(J_{0}\right)$ shown in Fig. प(b). The integral $J_{0}$ is finite, and the coefficients of its expansion in $\epsilon$, where $D=4-2 \epsilon$ is the dimensionality of space-time, have only one level of trancendentality [18], i.e., they contain poly-logarithms $\operatorname{Li}_{k}$ and zeta functions $\zeta(k)$ with the same value of $k$. These properties reduce the number of terms and thus simplify the calculation. In order to evaluate $J_{0}$, we temporarily introduce an artificial mass splitting among the four massive lines in Fig. (1) (b), by assigning the mass $m$ to any two of them and the mass $M$ to the other two. We then perform an expansion in the ratio $x=m^{2} / M^{2}$ using the large-mass expansion technique and recover the complete series in $x$ as explained in Ref. [18]. Through $O\left(\epsilon^{2}\right)$, we have

$$
\begin{aligned}
(1 & -\epsilon)\left(m^{2} M^{2}\right)^{2 \epsilon} m^{2} J_{0}=\sum_{n=1}^{\infty} x^{n}\left\{-\frac{8}{(2 n-1)^{3}}\right. \\
& +16 \epsilon\left[-\frac{\zeta(3)-\tilde{S}_{3}}{2 n-1}+\frac{2 \tilde{S}_{2}}{(2 n-1)^{2}}+\frac{4 \tilde{S}_{1}}{(2 n-1)^{3}}\right] \\
& +16 \epsilon^{2}\left[\frac{9 \zeta(4)+8 \zeta(3) \tilde{S}_{1}-4 \tilde{S}_{2}^{2}-8 \tilde{S}_{1} \tilde{S}_{3}-3 \tilde{S}_{4}}{2 n-1}\right. \\
& +\frac{4 \zeta(3)-16 \tilde{S}_{1} \tilde{S}_{2}-4 \tilde{S}_{3}}{(2 n-1)^{2}}-\frac{\zeta(2)+16 \tilde{S}_{1}^{2}+4 \tilde{S}_{2}}{(2 n-1)^{3}} \\
& \left.\left.-\frac{2}{(2 n-1)^{5}}\right]+O\left(\epsilon^{3}\right)\right\},
\end{aligned}
$$

where we introduced the short-hand notation $\tilde{S}_{a}=2^{a-2} S_{a}(2 n-1)-S_{a}(n-1)$, with $S_{a}(n)=$ $\sum_{j=1}^{n} j^{-a}$ being harmonic sums, and omitted irrelevant terms involving $\ln x$. We then put 
$x=1$ and exploit the identities

$$
\begin{aligned}
\sum_{n=1}^{\infty} \frac{\tilde{S}_{a}}{(2 n-1)^{c}}= & -\frac{2^{a}}{8} \sum_{l=1}^{\infty} \frac{1-(-1)^{l}}{l^{c}}\left[S_{a}(l)+2 S_{-a}(l)\right] \\
\sum_{n=1}^{\infty} \frac{\tilde{S}_{a} \tilde{S}_{b}}{(2 n-1)^{c}}= & \frac{2^{a+b}}{32} \sum_{l=1}^{\infty} \frac{1-(-1)^{l}}{l^{c}}\left[S_{a}(l)+2 S_{-a}(l)\right] \\
& \times\left[S_{b}(l)+2 S_{-b}(l)\right],
\end{aligned}
$$

at trancendentality levels $k=a+c$ and $k=a+b+c$, respectively. The sums with $k<5$ may be found in Ref. [18], while those with $k=5$ may be obtained from there through integration,

$$
\sum_{l=1}^{\infty} \frac{f(l) x^{l}}{l^{c+1}}=\int_{0}^{x} \frac{d y}{y} \sum_{l=1}^{\infty} \frac{f(l) y^{l}}{l^{c}},
$$

where $f(l)=S_{ \pm a}(l), \ldots$. After some algebra, we find an analytic expression for $J_{0}$ and hence also for $X_{0}$,

$$
X_{0}=-318 \zeta(4) \ln 2+\frac{873}{2} \zeta(5)-48 b_{5}+O(\epsilon) .
$$

Here and in the following, we use the constants

$$
\begin{aligned}
& a_{4}=\left(-2 \zeta(2)+\frac{\ln ^{2} 2}{3}\right) \ln ^{2} 2+8 \operatorname{Li}_{4}\left(\frac{1}{2}\right), \\
& a_{5}=\frac{1}{3}\left(2 \zeta(2)-\frac{\ln ^{2} 2}{5}\right) \ln ^{3} 2+8 \operatorname{Li}_{5}\left(\frac{1}{2}\right) .
\end{aligned}
$$

If we measure the matching scale $\bar{\mu}$ in units of the $\overline{\mathrm{MS}}$ mass $m_{h}(\bar{\mu})$, our result for the ratio of $a^{\prime}=a^{\left(n_{l}\right)}(\bar{\mu})$ to $a=a^{\left(n_{f}\right)}(\bar{\mu})$ reads

$$
\begin{aligned}
\frac{a^{\prime}}{a} & =1-a \frac{\ell}{6}+a^{2}\left(\frac{\ell^{2}}{36}-\frac{11}{24} \ell+c_{2}\right)+a^{3}\left[-\frac{\ell^{3}}{216}\right. \\
& \left.+\ell^{2}\left(\frac{53}{576}-\frac{n_{l}}{36}\right)+\ell\left(-\frac{955}{576}+\frac{67}{576} n_{l}\right)+c_{3}\right] \\
& +a^{4}\left\{\frac{\ell^{4}}{1296}+\ell^{3}\left(-\frac{1883}{10368}-\frac{127}{5184} n_{l}+\frac{n_{l}^{2}}{324}\right)\right. \\
& +\ell^{2}\left(\frac{2177}{3456}-\frac{1483}{10368} n_{l}-\frac{77}{20736} n_{l}^{2}\right)+\ell\left[\frac{7391699}{746496}\right. \\
& -\frac{2529743}{165888} \zeta(3)+n_{l}\left(-\frac{110341}{373248}+\frac{110779}{82944} \zeta(3)\right) \\
& \left.\left.+\frac{6865}{186624} n_{l}^{2}\right]+c_{4}\right\}+O\left(a^{5}\right),
\end{aligned}
$$




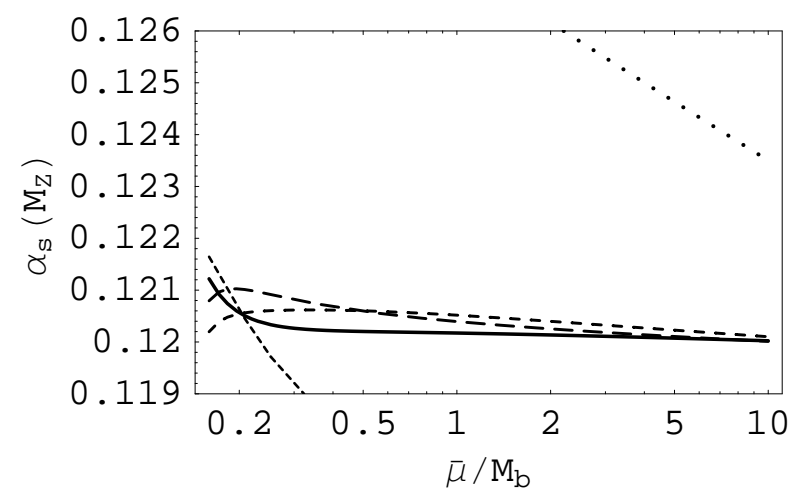

FIG. 2: $\bar{\mu}$ dependence of $\alpha_{s}^{(5)}\left(M_{Z}\right)$ from $N$-loop evolution and $(N-1)$-loop matching, with $N=1$ (dotted), 2 (short-dashed), 3 (dot-dashed), 4 (long-dashed), and 5 (solid).

where $\ell=\ln \left[\bar{\mu}^{2} / m_{h}^{2}(\bar{\mu})\right]$ and

$$
\begin{aligned}
& c_{2}=\frac{11}{72}, \quad c_{3}=\frac{564731}{124416}-\frac{82043}{27648} \zeta(3)-\frac{2633}{31104} n_{l}, \\
& c_{4}=\frac{291716893}{6123600}-\frac{2362581983}{87091200} \zeta(3)-\frac{76940219}{2177280} \zeta(4) \\
& +\frac{9318467}{362880} \zeta(4) \ln 2-\frac{12057583}{483840} \zeta(5)+\frac{3031309}{435456} a_{4} \\
& +\frac{340853}{90720} a_{5}+n_{l}\left(-\frac{4770941}{2239488}+\frac{3645913}{995328} \zeta(3)\right. \\
& \left.-\frac{541549}{165888} \zeta(4)+\frac{115}{576} \zeta(5)+\frac{685}{41472} a_{4}\right) \\
& +n_{l}^{2}\left(-\frac{271883}{4478976}+\frac{167}{5184} \zeta(3)\right) .
\end{aligned}
$$

The counterpart of Eq. (10) in the on-shell scheme of mass renormalization may be obtained by substituting the three-loop relationship between $m_{h}(\mu)$ and the pole mass $M_{h}$ [19].

Going to higher orders, one expects, on general grounds, that the relationship between $\alpha_{s}^{\left(n_{l}\right)}\left(\mu^{\prime}\right)$ and $\alpha_{s}^{\left(n_{f}\right)}(\mu)$, where $\mu^{\prime} \ll \bar{\mu} \ll \mu$, becomes insensitive to the choice of $\bar{\mu}$ as long as $\bar{\mu}=\mathcal{O}\left(m_{h}\right)$. This has been checked in Ref. [12] for four-loop evolution in connection with three-loop matching. Armed with our new results, we are in a position to explore the situation at the next order. As an example, we consider the crossing of the bottomquark threshold. In particular, we wish to study how the $\bar{\mu}$ dependence of the relationship between $\alpha_{s}^{(4)}\left(M_{\tau}\right)$ and $\alpha_{s}^{(5)}\left(M_{Z}\right)$ is reduced as we implement five-loop evolution with fourloop matching. Our procedure is as follows. We first calculate $\alpha_{s}^{(4)}(\bar{\mu})$ with Eq. (41) by imposing the condition $\alpha_{s}^{(4)}\left(M_{\tau}\right)=0.34[1]$, then obtain $\alpha_{s}^{(5)}(\bar{\mu})$ from the on-shell version 
of Eq. (101) with $M_{b}=4.85 \mathrm{GeV}[1]$, and finally compute $\alpha_{s}^{(5)}\left(M_{Z}\right)$ with Eq. (44). For consistency, $N$-loop evolution must be accompanied by $(N-1)$-loop matching, i.e., if we omit terms of $\mathcal{O}\left(1 / L^{N+1}\right)$ in Eq. (44), we need to discard those of $\mathcal{O}\left(a^{N}\right)$ in Eq. (10) at the same time. In Fig. 2, the variation of $\alpha_{s}^{(5)}\left(M_{Z}\right)$ with $\bar{\mu} / M_{b}$ is displayed for the various levels of accuracy, ranging from one-loop to five-loop evolution. For illustration, $\bar{\mu}$ is varied rather extremely, by almost two orders of magnitude. While the leading-order result exhibits a strong logarithmic behavior, the analysis is gradually getting more stable as we go to higher orders. The five-loop curve is almost flat. Besides the $\bar{\mu}$ dependence of $\alpha_{s}^{(5)}\left(M_{Z}\right)$, also its absolute normalization is significantly affected by the higher orders. At the central scale $\bar{\mu}=M_{b}$, we encounter an alternating convergence behavior.

As we have learned from Fig. 2, in higher orders, the actual value of $\bar{\mu}$ does not matter as long as it is comparable to the heavy-quark mass. In the context of Eq. (10), the choice $\bar{\mu}=\mu_{h}$, where $\mu_{h}=m_{h}\left(\mu_{h}\right)$ is the renormalization-group (RG) invariant $\overline{\mathrm{MS}}$ mass, is particularly convenient, since it eliminates the RG logarithm $\ell$. With this convention, we obtain from Eqs. (3), (41), and (10) a simple relationship between $\Lambda^{\prime}=\Lambda^{\left(n_{l}\right)}$ and $\Lambda=\Lambda^{\left(n_{f}\right)}$, viz

$$
\begin{aligned}
\beta_{0}^{\prime} & \ln \frac{\Lambda^{\prime 2}}{\Lambda^{2}}=\left(\beta_{0}^{\prime}-\beta_{0}\right) l+\left(b_{1}^{\prime}-b_{1}\right) \ln l-b_{1}^{\prime} \ln \frac{\beta_{0}^{\prime}}{\beta_{0}} \\
& +\frac{1}{\beta_{0} l}\left[b_{1}\left(b_{1}^{\prime}-b_{1}\right) \ln l+b_{1}^{\prime 2}-b_{1}^{2}-b_{2}^{\prime}+b_{2}+c_{2}\right] \\
& +\frac{1}{\left(\beta_{0} l\right)^{2}}\left[\frac{b_{1}^{3}}{2}\left(\ln ^{2} l-1\right)-b_{1}^{\prime} b_{1}^{2}\left(\frac{1}{2} \ln ^{2} l-\ln l-1\right)\right. \\
& -b_{1}\left(b_{1}^{\prime 2}-b_{2}^{\prime}+b_{2}+c_{2}\right) \ln l-\frac{b_{1}^{\prime 3}}{2}+b_{1}^{\prime}\left(b_{2}^{\prime}-b_{2}-c_{2}\right) \\
& \left.-\frac{1}{2}\left(b_{3}^{\prime}-b_{3}\right)+c_{3}\right]+\frac{1}{\left(\beta_{0} l\right)^{3}}\left\{-b_{1}^{4}\left(\frac{1}{3} \ln ^{3} l-\frac{1}{2} \ln ^{2} l\right.\right. \\
& \left.-\ln l-\frac{1}{6}\right)+b_{1}^{\prime} b_{1}^{3}\left(\frac{1}{3} \ln ^{3} l-\frac{3}{2} \ln { }^{2} l-\ln l+\frac{1}{2}\right)+b_{1}^{2} \\
& \times\left(b_{1}^{\prime 2}-b_{2}^{\prime}+b_{2}+c_{2}\right)\left(\ln ^{2} l-\ln l-1\right)+b_{1}\left[b_{1}^{\prime 3}-2 b_{1}^{\prime}\right. \\
& \left.\times\left(b_{2}^{\prime}-b_{2}-c_{2}\right)+b_{3}^{\prime}-b_{3}-2 c_{3}\right] \ln l+\frac{b_{1}^{\prime 4}}{3}-b_{1}^{\prime 2}\left(b_{2}^{\prime}\right. \\
& \left.-b_{2}-c_{2}\right)+\left(b_{2}^{\prime}-b_{2}\right)\left(\frac{b_{2}^{\prime}}{3}-\frac{2}{3} b_{2}-c_{2}\right)-c_{2}^{2}+b_{1}^{\prime}\left(\frac{2}{3}\right. \\
& \left.\left.\times b_{3}^{\prime}-\frac{b_{3}}{2}-c_{3}\right)-\frac{b_{1} b_{3}}{6}-\frac{1}{3}\left(b_{4}^{\prime}-b_{4}\right)+c_{4}\right\}+O\left(\frac{1}{l^{4}}\right)
\end{aligned}
$$

where $l=\ln \left(\mu_{h}^{2} / \Lambda^{2}\right)$. The $\mathcal{O}\left(1 / l^{3}\right)$ term of Eq. (12) is new. Equation (12) represents a 
closed four-loop formula for $\Lambda^{\left(n_{l}\right)}$ in terms of $\Lambda^{\left(n_{f}\right)}$ and $\mu_{h}$. For consistency, it should be

used in connection with the five-loop expression (4) for $\alpha_{s}^{\left(n_{f}\right)}(\mu)$ with the understanding that the underlying flavor thresholds are fixed at $\bar{\mu}=\mu_{h}$. The inverse relation that gives $\Lambda^{\left(n_{f}\right)}$ as a function of $\Lambda^{\left(n_{l}\right)}$ and $\mu_{h}$ emerges from Eq. (12) via the substitutions $\Lambda \leftrightarrow \Lambda^{\prime} ; \beta_{N} \leftrightarrow \beta_{N}^{\prime}$ for $N=0, \ldots, 4$; and $c_{N} \rightarrow-c_{N}$ for $N=2,3,4$.

In conclusion, we have extended the standard description of the strong-coupling constant in the $\overline{\mathrm{MS}}$ renormalization scheme to include five-loop evolution and four-loop matching at the flavor thresholds. These results will be indispensable in order to relate the QCD predictions for different observables at next-to-next-to-next-to-next-to-leading order.

\section{Acknowledgments}

We thank J.A.M. Vermaseren for a useful communication regarding Ref. 9]. A.V.K. was supported in part by the RFBR Foundation through Grant No. 05-02-17645-a and by the Heisenberg-Landau-Programm. This work was supported in part by BMBF Grant No. 05 HT4GUA/4 and HGF Grant No. NG-VH-008.

[1] Particle Data Group, S. Eidelman et al., Phys. Lett. B 592, 1 (2004); URL: http://pdg.lbl.gov/

[2] D.J. Gross and F. Wilczek, Phys. Rev. Lett. 30, 1343 (1973); H.D. Politzer, ibid. 30, 1346 (1973).

[3] C.G. Bollini and J.J. Giambiagi, Phys. Lett. 40B, 566 (1972); G. 't Hooft and M. Veltman, Nucl. Phys. B44, 189 (1972); G. 't Hooft, ibid. B61, 455 (1973).

[4] D.R.T. Jones, Nucl. Phys. B75, 531 (1974); W.E. Caswell, Phys. Rev. Lett. 33, 244 (1974); É.Sh. Egoryan and O.V. Tarasov, Teor. Mat. Fiz. 41, 26 (1979) [Theor. Math. Phys. 41, 863 (1979)].

[5] O.V. Tarasov, A.A. Vladimirov, and A.Yu. Zharkov, Phys. Lett. 93B, 429 (1980); S.A. Larin and J.A.M. Vermaseren, ibid. B 303, 334 (1993).

[6] T. van Ritbergen, J.A.M. Vermaseren, and S.A. Larin, Phys. Lett. B 400, 379 (1997).

[7] W.A. Bardeen, A.J. Buras, D.W. Duke, and T. Muta, Phys. Rev. D 18, 3998 (1978). 
[8] J.A. Gracey, Phys. Lett. B 373, 178 (1996).

[9] J. Ellis, I. Jack, D.R.T. Jones, M. Karliner, and M.A. Samuel, Phys. Rev. D 57, 2665 (1998).

[10] T. Appelquist and J. Carazzone, Phys. Rev. D 11, 2856 (1975).

[11] W. Bernreuther and W. Wetzel, Nucl. Phys. B197, 228 (1982); B513, 758(E) (1998); S.A. Larin, T. van Ritbergen, and J.A.M. Vermaseren, ibid. B438, 278 (1995).

[12] K.G. Chetyrkin, B.A. Kniehl, and M. Steinhauser, Phys. Rev. Lett. 79, 2184 (1997); Nucl. Phys. B510, 61 (1998).

[13] Y. Schröder and M. Steinhauser, JHEP 0601, 051 (2006); K.G. Chetyrkin, J.H. Kühn, and C. Sturm, Nucl. Phys. B744, 121 (2006).

[14] Y. Schroder and A. Vuorinen, JHEP 0506, 051 (2005); K.G. Chetyrkin, M. Faisst, C. Sturm, and M. Tentyukov, Nucl. Phys. B742, 208 (2006).

[15] K.G. Chetyrkin, J.H. Kühn, C. Sturm, Report No. SFB/CPP-06-21, TTP06-15, hep-ph/0604234 R. Boughezal, M. Czakon, T. Schutzmeier, Report No. hep-ph/0605023 R. Boughezal and M. Czakon, Report No. hep-ph/0606232

[16] B.A. Kniehl and A.V. Kotikov, Phys. Lett. B 638, 531 (2006); Report No. DESY 06-073.

[17] W. Furmanski and R. Petronzio, Z. Phys. C 11, 293 (1982).

[18] J. Fleischer, A.V. Kotikov, and O.L. Veretin, Nucl. Phys. B547, 343 (1999).

[19] K. Melnikov and T. van Ritbergen, Phys. Lett. B 482, 99 (2000). 